\title{
An Extremely Rare Case of Splenic Rupture Secondary to Metastatic Gestational Choriocarcinoma
}

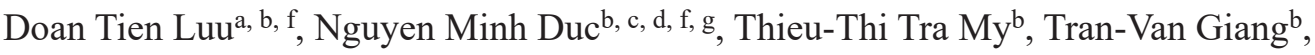 \\ Luong Viet Bange, Bui-Van Lenha, ${ }^{\mathrm{a}}$
}

\begin{abstract}
Gestational choriocarcinoma (GC) is an uncommon malignant tumor consisting of trophoblastic cells. The lungs, liver, and central nervous system are the most common metastatic sites for this disease. However, splenic metastasis is unusual and might result in spontaneous rupture. Symptoms associated with splenic rupture may be the first presentation of malignancy. A thorough medical history and examination are necessary to detect the primary lesion. Herein, we present a case of a 23-year-old female who had splenic rupture secondary to choriocarcinoma metastasis. Although the emergency condition had been solved, the patient died 1 month after due to brain metastasis. The goal of this article was to report a new case of spontaneous splenic rupture caused by choriocarcinoma metastasis and to review the existing literature on splenic metastases associated with GC, including the epidemiology and etiology.
\end{abstract}

Keywords: Choriocarcinoma; Gestational trophoblastic neoplasm; Splenic metastasis; Splenic rupture

\section{Introduction}

Gestational choriocarcinoma (GC), a malignant gestational trophoblastic neoplasm (GTN) representing a trimorphic proliferation of intermediate trophoblastic cells, syncytiotrophoblasts, and cytotrophoblasts [1], has an incidence of approxi-

Manuscript submitted January 12, 2021, accepted February 1, 2021

Published online February 10, 2021

aDepartment of Radiology, Ha Noi Medical University Hospital, Ha Noi, Vietnam

bDepartment of Radiology, Ha Noi Medical University, Ha Noi, Vietnam 'Department of Radiology, Pham Ngoc Thach University of Medicine, Ho Chi Minh City, Vietnam

dDepartment of Radiology, Children's Hospital 2, Ho Chi Minh City, Vietnam 'Department of Pathology, Tam Anh General Hospital, Ha Noi, Vietnam

${ }^{f}$ These authors contributed equally as co-first authors.

gCorresponding Author: Nguyen Minh Duc, Department of Radiology, Pham Ngoc Thach University of Medicine, Ho Chi Minh City, Vietnam.

Email: bsnguyenminhduc@pnt.edu.vn

doi: https://doi.org/10.14740/wjon1356 mately 1 in 50,000 pregnancies [2]. GC can metastasize to the brain, lungs, liver, kidney, and vagina [3], but splenic metastasis represents fewer than 5\% of cases [4]. GTNs are highly vascular, and severe spontaneous hemorrhage can occur in response to tumor biopsy [1]. Metastatic lesion characteristics are similar to those of primary tumors, and spontaneous secondary tumor rupture has been reported in the brain, spleen, and liver $[5,6]$. Patients with splenic metastasis may present with acute abdomen due to spontaneous splenic rupture [7].

\section{Case Report}

A 23-year-old female presented with sudden-onset left upper quadrant pain for $2 \mathrm{~h}$. Her presentation was as follows: pulse rate, $100 \mathrm{bpm}$ in sinus rhythm; hypotensive to $90 / 60$ $\mathrm{mm} \mathrm{Hg}$; temperature, $37{ }^{\circ} \mathrm{C}$; respiratory rate, 18 breaths per min, with $91 \%$ saturation on room air; tenderness in the left upper abdomen; and minimal cervical blood on vaginal examination. She had no history of recent trauma and was gravida 2, para 2, with full-term delivery and prolonged vaginal bleeding 5 weeks before symptom onset. Complete blood count showed: low hemoglobin, $8 \mathrm{~g} / \mathrm{dL}$; white cell count, $7,000 / \mathrm{mm}^{3}$; and platelet count, $170,000 / \mathrm{mm}^{3}$, with normal coagulation profile. Her electrolytes, blood urea nitrogen, creatinine, lipase, total bilirubin, alanine aminotransferase, aspartate aminotransferase, and urinalysis were normal. An abdominal pre-contrast computed tomography (CT) revealed free intraperitoneal fluid, with hyperdense fluid (70 Hounsfield unit (HU)), surrounding the spleen (Fig. 1). Blood was detected in the peritoneal cavity. On the arterial phase, a 3 $\times 4 \mathrm{~cm}$ splenic mass was observed (Fig. 2a), which showed heterogeneous enhancement and was hypervascular, with illdefined margins (Fig. 2a, b). The mass showed heterogeneous enhancement in the venous phase (Fig. 2c). A tumor laceration on the splenic capsule was noted (Fig. 2a, c). Vascular injury appeared as a focal collection of vascular contrast on the arterial phase, with decreased attenuation on the venous phase (Fig. 2b, d). A $1.5 \times 2 \mathrm{~cm}$ hypoenhancing mass was identified on the anterior uterine wall (Fig. 3). This patient was diagnosed with ruptured splenic mass leading to hemoperitoneum, and emergency splenectomy was performed. Histopathology results revealed a solid tumor (Fig. 4a), with hemorrhage and necrosis (Fig. 4b). Diffusely infiltrative trimorphic malignant trophoblasts, rimmed by multinucleated 

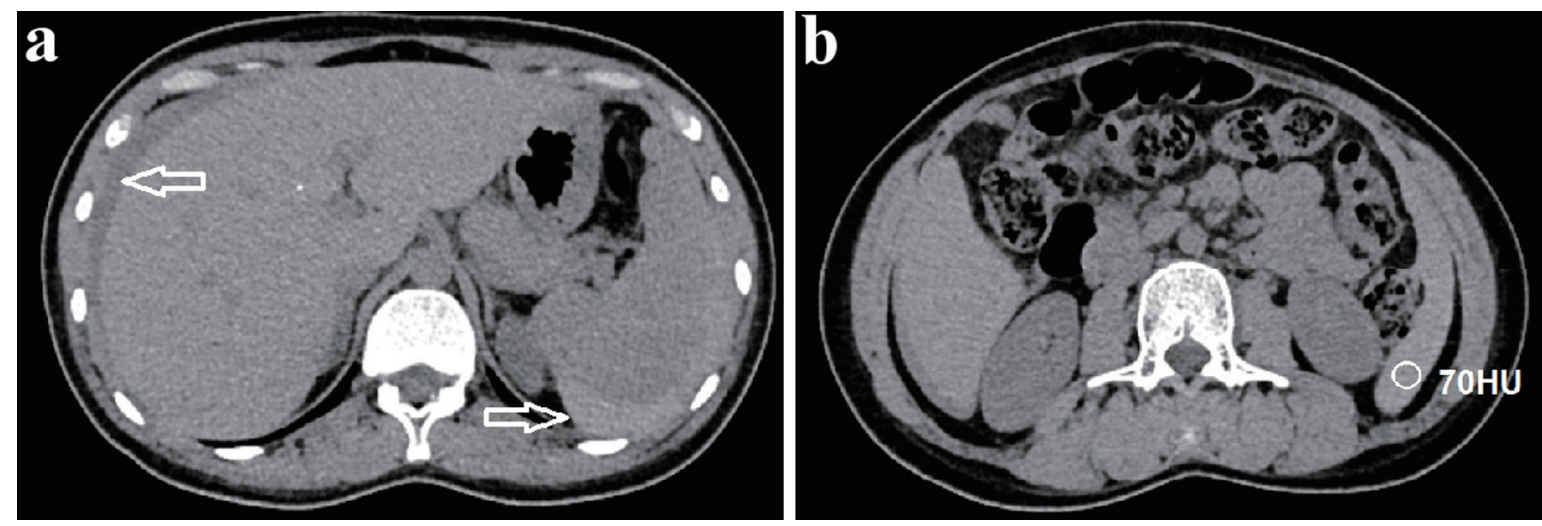

Figure 1. Axial abdominal CT pre-contrast images. (a) Free intraperitoneal fluid (arrow) was detected. (b) The fluid surrounding the spleen was hyperdense, measuring 70 Hounsfield unit $(\mathrm{HU})$, suggesting blood within the peritoneal cavity. CT: computed tomography.

syncytiotrophoblasts (Fig. 4c, d) were observed. Marked cytologic atypia and mitotic figures were present (Fig. 4c, d). Immunohistochemically, the neoplastic syncytiotrophoblastic cells showed strong and diffuse positivity for cytokeratins (CK), cytokeratin 7 (CK7), human chorionic gonadotropin (HCG), placental alkaline phosphatase (PLAP), and inhibin, with a Ki-67 index above $90 \%$ (Fig. 5). The final diagnosis was splenic rupture secondary to GC metastasis. The patient died after 1 month due to brain metastasis.

\section{Discussion}

As a rare and aggressive form of GTN [1], approximately $50 \%$ of GC cases develop from complete hydatidiform moles, $25 \%$ develop following miscarriage or ectopic pregnancy, and $25 \%$
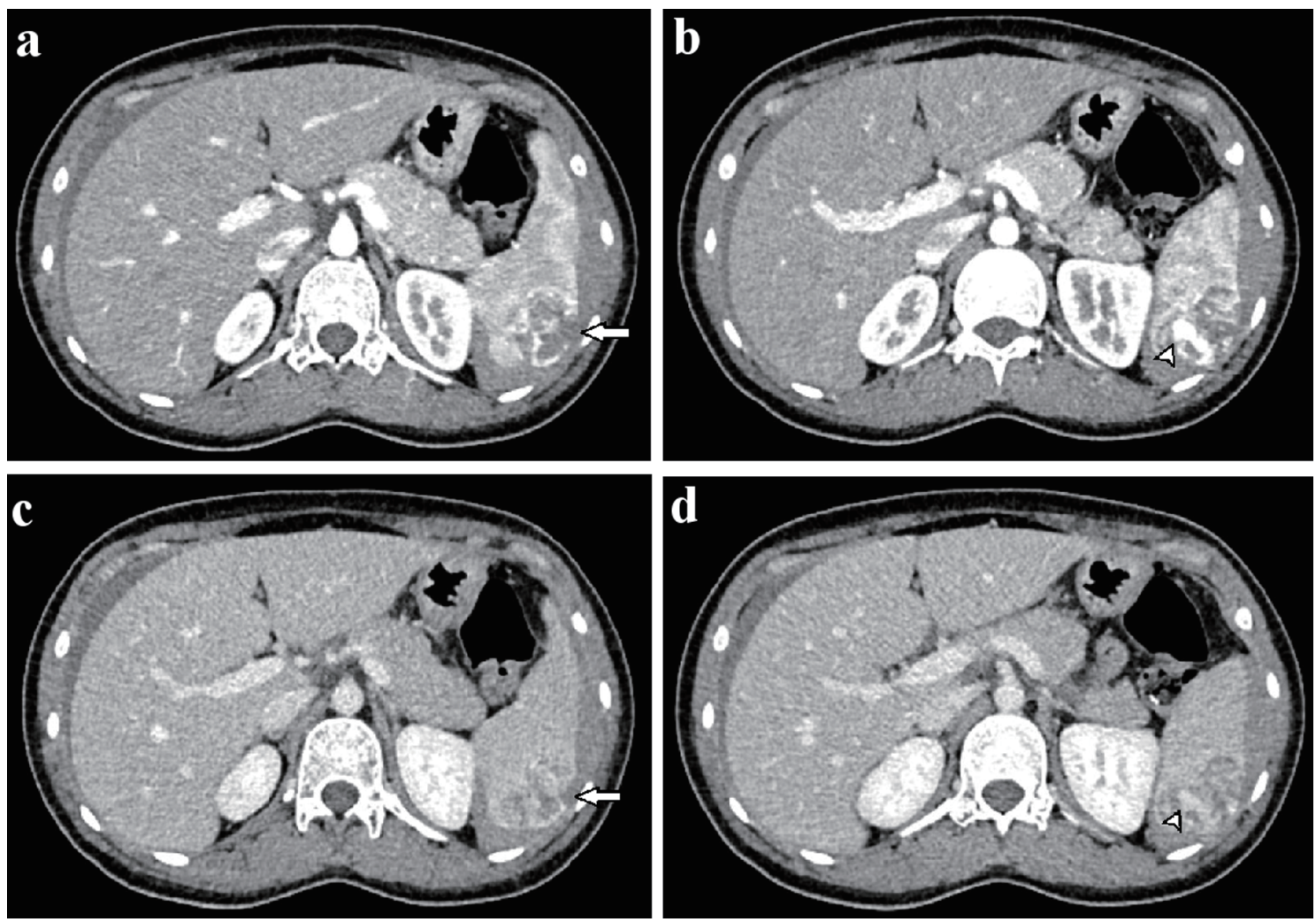

Figure 2. Axial abdominal CT in the arterial $(a, b)$ and venous phases (c, d). A $3 \times 4 \mathrm{~cm}$ mass with ill-defined borders was observed in the spleen (a). This mass showed heterogeneous enhancement and hypervascularity ( $a, b)$. The mass enhanced heterogeneously on the venous phase (c). A tumor laceration was observed on the splenic capsule (a, c), resulting in intratumor pseudoaneurysm (b, d; arrowhead). CT: computed tomography. 


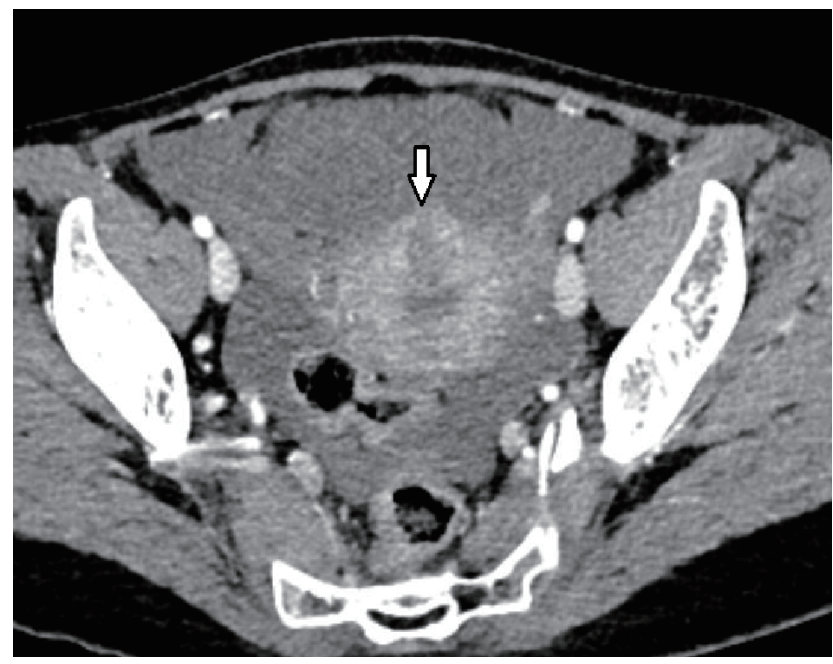

Figure 3. A hypoenhancing mass was observed on the uterine wall.

develop after full-term pregnancy [8]. Approximately $80 \%$ of GTNs feature metastatic lesions in the lungs $(80 \%)$, vagina $(30 \%)$, pelvis $(20 \%)$, liver $(10 \%)$, and brain $(10 \%)$ [9], with splenic metastasis incidence below 5\% [4]. These highly vascular tumors are prone to aneurysms [5]. Splenic GC rupture can cause heavy bleeding and is rare, with Olivier et al identifying fewer than 10 cases [10]. Patients with splenic metastasis often experience acute abdomen, characterized by severe tenderness in response to palpation in the left upper quadrant, rigidity, or abdominal distention [11]. Severe patients may present with hypovolemic shock, tachycardia, or hypotension [12]. Patients may also present with primary tumor symptoms, including uterine bleeding, anemia, and hyperemesis [1]. Splenic tumor can be observed on CT with contrast enhancement. Splenic artery pseudoaneurysm, active bleeding, or splenic lacerations associated with hemoperitoneum may present [7]. In histopathological assessments, GCs present as diffusely infiltrative, solid masses of trimorphic malignant trophoblasts [13]. Tumors consist of intermediate trophoblasts, cytotrophoblasts, and syncytiotrophoblasts, with numerous cytological atypia and mitotic figures [14]. Hemorrhage and necrosis are common [14]. On immunohistochemical analysis, tumor cells were strongly, diffusely HCG-positive, positive for CK, CK7, inhibin, and PLAP, and $>90 \%$ Ki67-positive $[13,14]$, supporting GC diagnosis [14]. Following splenic GC rupture, emergency surgery or embolization are utilized to stop bleeding $[7,15$, 16]. Multi-agent chemotherapy, including etoposide, actinomycin D, methotrexate, folinic acid, and cyclophosphamide, is typical treatment [8]. High-risk patients score above 7 on the International Federation of Gynecology and Obstetrics (FIGO)
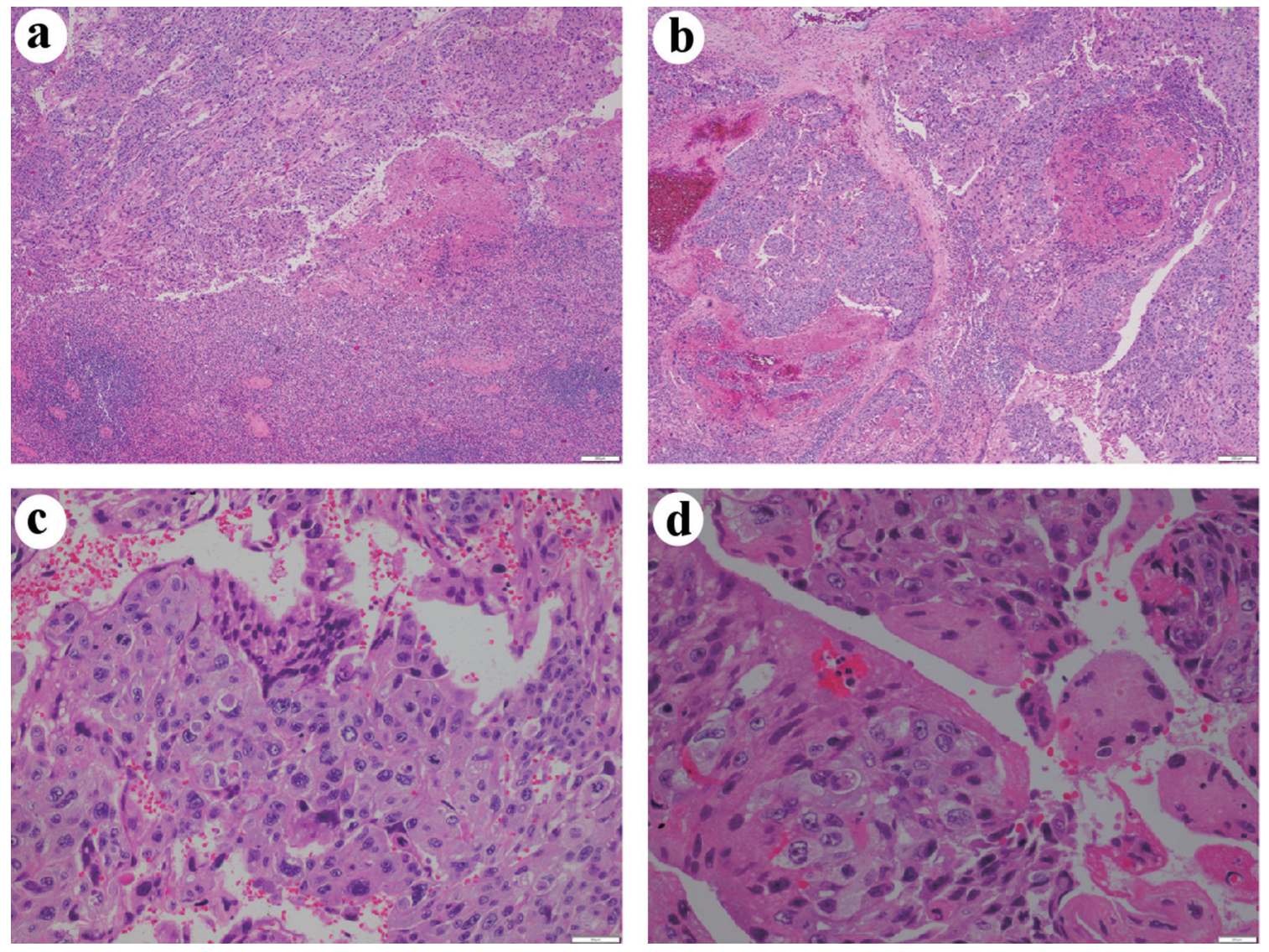

Figure 4. Hematoxylin and eosin (H\&E) staining. (a) The tumor was solid, with large dyscohesive and pleomorphic cells $(\times 40)$. (b) Hemorrhagic and necrotic areas were observed $(\times 40)$. (c) Two types of identified cells: oval and giant cells, similar to cytotrophoblastic cells and syncytiotrophoblastic cells, respectively ( $\times 200)$. (d) Cytologic atypia and mitotic figures $(\times 400)$. 

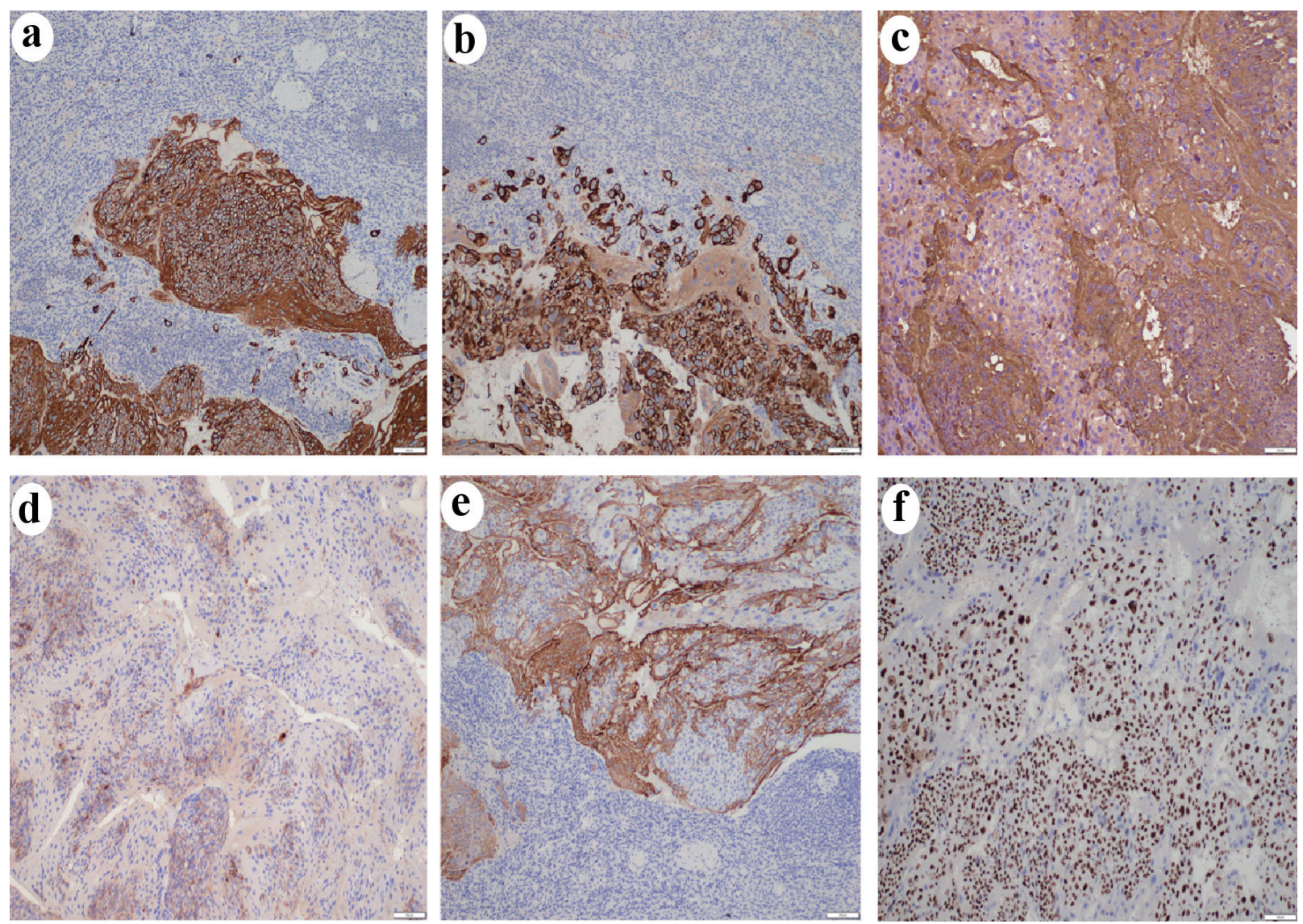

Figure 5. Immunohistochemistry results $(\times 100)$ showed tumor cells positive for CK (a), CK7 (b), HCG (c), PLAP (d), and inhibin (e). A high Ki-67 labeling index (> 90\%) was observed (f). CK: cytokeratins; CK7: cytokeratin 7; HCG: human chorionic gonadotropin; PLAP: placental alkaline phosphatase.

scoring system for GTN [17].

Age and term pregnancy history are GC risk factors. Because splenic metastasis is very rare, the possibility of metastatic tumor was not sufficiently explored. This patient experienced GC after a term pregnancy, with multiple metastases, including brain metastasis, suggesting high-risk disease.

\section{Conclusions}

GC is a rare and aggressive type of gestational trophoblastic neoplasia. Metastatic GC resulting in atraumatic splenic rupture is rare. Spontaneous splenic rupture without trauma indicates pathological rupture. This diagnosis should be considered in postpartum women with irregular vaginal bleeding and acute abdomen. A total splenectomy can facilitate a definitive diagnosis and avoid complications. Unfortunately in this patient, despite having a splenectomy, the patient still died from brain metastases.

\section{Acknowledgments}

None to declare.

\section{Financial Disclosure}

None to declare.

\section{Conflict of Interest}

The authors do not have any conflict of interest.

\section{Informed Consent}

Written informed consent of patient was obtained.

\section{Author Contributions}

Conceptualization: Doan TL and Nguyen MD; data curation: Thieu-Thi TM and Nguyen MD; formal analysis: Doan TL and Nguyen MD; writing of original draft: Nguyen MD and ThieuThi TM; writing of review and editing: Doan TL, Thieu-Thi $\mathrm{TM}$, and Nguyen MD; All authors have read and agreed to the published version of the manuscript. 


\section{Data Availability}

The authors declare that data supporting the findings of this study are available within the article.

\section{References}

1. Stevens FT, Katzorke N, Tempfer C, Kreimer U, Bizjak GI, Fleisch MC, Fehm TN. Gestational trophoblastic disorders: an update in 2015. Geburtshilfe Frauenheilkd. 2015;75(10):1043-1050.

2. Ngan S, Seckl MJ. Gestational trophoblastic neoplasia management: an update. Curr Opin Oncol. 2007;19(5):486-491.

3. Cheng H, Yang J, Ren T, Zhao J, Feng F, Wan X, Xiang Y. Gestational trophoblastic neoplasia with urinary system metastasis: a single center experience. Front Oncol. 2020;10:1208.

4. Ashraf-Ganjooei T, Ghaemmaghami F. Patients with presenting unusual manifestations with gestational trophoblastic neoplasm: case series and review of literatures. Arch Gynecol Obstet. 2008;277(5):465-470.

5. Giannakopoulos G, Nair S, Snider C, Amenta PS. Implications for the pathogenesis of aneurysm formation: metastatic choriocarcinoma with spontaneous splenic rupture. Case report and a review. Surg Neurol. 1992;38(3):236240.

6. Gulati A, Vyas S, Lal A, Harsha TS, Gupta V, Nijhawan R, Khandelwal N. Spontaneous rupture of hepatic metastasis from choriocarcinoma: a review of imaging and management. Ann Hepatol. 2009;8(4):384-387.

7. Galazi M, Tait P, Seckl M, Savage P. Successful embolisation of a life threatening bleeding splenic metastasis in a patient with gestational choriocarcinoma. Clin Obstet
Gynecol Reprod Med. 2015;1(1):16-18.

8. Goldstein DP, Berkowitz RS. Current management of gestational trophoblastic neoplasia. Hematol Oncol Clin North Am. 2012;26(1):111-131.

9. Berkowitz RS, Goldstein DP. Chorionic tumors. N Engl J Med. 1996;335(23):1740-1748.

10. Olivier M, Hugues IN, Joseph BN, et al. Gestational Choriocarcinoma of the uterus with concurrent metastasis and an atraumatic rupture of the spleen: a rare association and first description in Africa. Am J Obstet Gynecol Res. 2019;1(1):1002.

11. Weaver H, Kumar V, Spencer K, Maatouk M, Malik S. Spontaneous splenic rupture: A rare life-threatening condition; Diagnosed early and managed successfully. Am J Case Rep. 2013;14:13-15.

12. Gedik E, Girgin S, Aldemir M, Keles C, Tuncer MC, Aktas A. Non-traumatic splenic rupture: report of seven cases and review of the literature. World J Gastroenterol. 2008;14(43):6711-6716.

13. Gurzu S, Copotoiu C, Tugui A, Kwizera C, Szodorai R, Jung I. Primary gastric choriocarcinoma - a rare and aggressive tumor with multilineage differentiation: A case report. World J Clin Cases. 2019;7(14):1837-1843.

14. International Agency for Research on Cancer, World Health Organization. WHO classification of tumours of female reproductive organs. 4th ed. International Agency for Research on Cancer; 2014.

15. Nethra S, Hawe J, Elder J. Splenic rupture: a rare presentation of metastatic choriocarcinoma. Gynecol Surg. 2011;8(4):435-437.

16. Aloysius TMN, Shelat VG. Laparoscopic splenectomy for splenic rupture secondary to metastatic choriocarcinoma. Ann Hepatobiliary Pancreat Surg. 2018;22(1):7982.

17. Froeling FE, Seckl MJ. Gestational trophoblastic tumours: an update for 2014. Curr Oncol Rep. 2014;16(11):408. 